\title{
Automatic Improvements of Wallis' Inequality
}

\author{
Peter Paule \\ Research Institute for Symbolic Computation (RISC), \\ Johannes Kepler University, A-4040 Linz, AUSTRIA \\ Veronika Pillwein \\ Research Institute for Symbolic Computation (RISC), \\ Johannes Kepler University, A-4040 Linz, AUSTRIA
}

\begin{abstract}
The mathematical backbone of this article is formed by three classical formulas of Wallis: his product formula for $\pi$, an inequality implying the product formula in the limit, and a related definite integral involving powers of the sine function. For the latter we present various evaluations to illustrate recent algorithmic developments. In the main part of the article we automatically refine the bounds in Wallis' inequality by using methods based on Collins' cylindrical algebraic decomposition.
\end{abstract}

Key words: Wallis product formula, Wallis integral, symbolic integration, symbolic summation, cylindrical algebraic decomposition, inequalities

Dedicated to Keith Geddes on the occasion of his 60th birthday

\section{Introduction}

The types of mathematics being considered in this article are related to some of Keith Geddes' research interests, namely: computational aspects of algebra and analysis, including the solution of problems in integral and differential calculus, and closed-form summation ${ }^{1}$. The mathematical backbone of our study is formed by three classical results of John Wallis (12).

\footnotetext{
^ The second named author was supported by grant P20162 of the Austrian Science Foundation FWF. Email addresses: ppaule@risc.uni-linz.ac.at (Peter Paule), vpillwei@risc.uni-linz.ac.at (Veronika Pillwein).

1 This sentence is a slight variation of a statement from Keith Geddes' home page at http://www.cs.uwaterloo.ca/ kogeddes/
} 
Theorem 1 (Wallis' product formula).

$$
\frac{\pi}{2}=\frac{2}{1} \frac{2}{3} \frac{4}{3} \frac{4}{5} \frac{6}{5} \frac{6}{7} \cdots
$$

Theorem 2 (Wallis' integral formula). For $m \in \mathbb{N}=\{0,1, \ldots\}$ let

$$
A(m):=\int_{0}^{\pi / 2} \sin (t)^{m} d t .
$$

Then

$$
A(2 n)=\frac{\pi}{2^{2 n+1}}\left(\begin{array}{c}
2 n \\
n
\end{array}\right) \quad \text { and } \quad A(2 n+1)=\frac{2^{2 n}}{2 n+1}\left(\begin{array}{c}
2 n \\
n
\end{array}\right)^{-1}, \quad n \geq 0 .
$$

Theorem 3 (Wallis' inequality). For $n \in \mathbb{N}$ let

$$
c(n):=\frac{2^{4 n+1}}{2 n+1}\left(\begin{array}{c}
2 n \\
n
\end{array}\right)^{-2} .
$$

Then

$$
\frac{2 n}{2 n+1} \leq \frac{c(n)}{\pi} \leq 1, \quad n \geq 0
$$

Wallis' product formula (1) is an immediate consequence of taking the limit $n \rightarrow \infty$ in Wallis' inequality (4). As explained in almost each introductory analysis text, Wallis' inequality (4) is a straight-forward consequence of Wallis' integral formula (2). Namely, for $t \in[0, \pi / 2]$ one has $\sin (t)^{2 n+2} \leq \sin (t)^{2 n+1} \leq \sin (t)^{2 n}$, which implies

$$
\frac{A(2 n+2)}{A(2 n)} \leq \frac{A(2 n+1)}{A(2 n)} \leq 1, \quad n \geq 0 .
$$

Observing that $A(2 n+2) / A(2 n)=(2 n+1) /(2 n+2)$ (by $(2))$ and $A(2 n+1) / A(2 n)=$ $c(n) / \pi$ we obtain

$$
\frac{2 n+1}{2 n+2} \leq \frac{c(n)}{\pi} \leq 1, \quad n \geq 0 .
$$

Note that $(5)$ due to $2 n /(2 n+1) \leq(2 n+1) /(2 n+2)$ can be viewed as a refinement of (4). In Section 3.2, the main part of this article, we will describe some ideas how to derive such improvements of inequalities in an algorithmic fashion.

Before coming to these aspects, we devote Section 2 to Wallis' integral formula (2) that, according to Moll (3, p.32), "is one of the first exact evaluations of a definite integral." Taking the opportunity to illustrate recent algorithmic progress with definite special function integrals, in Section 2 we will present several proofs of this classical key result (2). Above we have seen that (1) and (4) are consequences of it; further applications, and also proofs, can be found e.g. in (3).

Section 3 begins with a "short story" leading up to an inequality (15), originally conjectured by Moll in the context of Wallis' integral. Section 3.1 presents an elementary introduction to recent computer algebra developments for proving inequalities like (15). Finally, Section 3.2 is devoted to automatic refinements of bounds in Wallis' inequality (4). 


\section{Proving Wallis' Integral Formula}

The standard proof of the classical key result (2) is by deriving the recurrence

$$
(m+2) A(m+2)-(m+1) A(m)=0, \quad m \geq 0,
$$

with integration by parts. This together with the initial values $A(0)=\pi / 2$ and $A(1)=1$ proves (2). In order to illustrate recent algorithmic progress, we present some other proofs.

\subsection{Hypergeometric aspects}

Systems like Maple have no problem to return a closed form evaluation in terms of the gamma function, for instance,

$$
A(m)=\frac{1}{2} \frac{\Gamma\left(\frac{m+1}{2}\right)}{\Gamma\left(\frac{m+2}{2}\right)}, \quad m \in \mathbb{N} .
$$

However, the question about how this result has been derived might lead to ask the system for the indefinite integral. For example, when using Mathematica (V 6.0) one obtains that

$$
\int \sin (t)^{m} d t=-\cos (t){ }_{2} F_{1}\left(\begin{array}{c}
\frac{1}{2}, \frac{1-m}{2} \\
\frac{3}{2}
\end{array} ; \cos (t)^{2}\right),
$$

where the ${ }_{2} F_{1}$ expression is a hypergeometric series; for its definition see e.g. (2). Leaving aside the question about how to derive (8), the user learns from this representation that (7) can be obtained from it with Gauss' summation formula (2, Thm.2.2.2):

$$
{ }_{2} F_{1}\left(\begin{array}{c}
a, b \\
c
\end{array} ; 1\right)=\frac{\Gamma(c) \Gamma(c-a-b)}{\Gamma(c-a) \Gamma(c-b)}, \quad \operatorname{Re}(c-a-b)>0 .
$$

Remark. We note that (8) is a mere rewriting of the integral by means of Euler's integral representation for the hypergeometric function. With the substitution $s=\cos (t)^{2}$, i.e., $d s=-2 \sin (t) \cos (t) d t$, we obtain

$$
-\frac{1}{2} \int_{0}^{u}(1-s)^{(m-1) / 2} s^{-1 / 2} d s=\int_{\pi / 2}^{\arccos (\sqrt{u})} \sin (t)^{m} d t .
$$

Euler's transform (2, Thm.2.2.1) gives

$$
-u^{1 / 2}{ }_{2} F_{1}\left(\begin{array}{c}
\frac{1}{2}, \frac{1-m}{2} \\
\frac{3}{2}
\end{array} ;\right)=-\frac{u^{1 / 2}}{2} \int_{0}^{1} t^{-1 / 2}(1-u t)^{(m-1) / 2} d t=-\frac{1}{2} \int_{0}^{u} \frac{(1-s)^{(m-1) / 2}}{s^{-1 / 2}} d s .
$$

This together with the substitution $u=\cos (x)^{2}$ completes the proof of (8). 


\subsection{Evaluation by creative telescoping}

The definite integration problem can be transformed into a definite summation problem. Namely, for $m \in \mathbb{N}$ :

$$
\begin{aligned}
A(m) & =\int_{0}^{\pi / 2} \sin (t)^{m} d t=\int_{0}^{\pi / 2}\left(\frac{e^{i t}-e^{-i t}}{2 i}\right)^{m} d t \\
& =\left(\frac{1}{2 i}\right)^{m} \sum_{k=0}^{m}\left(\begin{array}{c}
m \\
k
\end{array}\right)(-1)^{k} \int_{0}^{\pi / 2} e^{(m-2 k) i t} d t .
\end{aligned}
$$

From the simple evaluation of the integral one immediately obtains for $n \in \mathbb{N}$ :

$$
A(2 n)=\frac{\pi}{2^{2 n+1}}\left(\begin{array}{c}
2 n \\
n
\end{array}\right)
$$

and

$$
A(2 n+1)=\frac{(-1)^{n}}{2^{2 n+1}} \sum_{k=0}^{2 n+1}\left(\begin{array}{c}
2 n+1 \\
k
\end{array}\right) \frac{(-1)^{k}}{2 n-2 k+1} .
$$

The task to simplify the definite sum in (9) is trivial by applying any implementation of Zeilberger's creative telescoping $((13),(14))$ which computes

$$
(2 n+3) A(2 n+3)-2(n+1) A(2 n+1)=0, \quad n \geq 0 .
$$

This recurrence together with the initial value $A(1)=1$ completes the proof of (2).

\subsection{Evaluation by a differential analogue to creative telescoping}

By a differential analogue to creative telescoping a recurrence for $A(m)$ can be computed in a direct fashion, i.e., without reformulating integration as a summation problem. Namely, let us consider the task to find polynomials $c_{i} \in \mathbb{Q}[m]$ together with a function $g_{m}(t)$ such that

$$
c_{0}(m) \sin (t)^{m+2}+c_{1}(m) \sin (t)^{m+1}+c_{2}(m) \sin (t)^{m}=\frac{d}{d t} g_{m}(t) .
$$

M. Kauers' "extended Risch package" (7), a combination of the Risch integration algorithm with a differential analogue of creative telescoping, computes:

$$
c_{0}(m)=m+2, c_{1}(m)=0, c_{2}(m)=-(m+1), \text { and } g_{m}(t)=-\frac{1}{2} \sin (2 t) \sin (t)^{m} .
$$

Using these values, taking for both sides of (11) the integral from 0 to $\pi / 2$, gives the recurrence (6).

Remark. The Wallis integral $A(m)$ is not in the scope of the Almkvist-Zeilberger algorithm (1), since $\sin (t)^{m}$ is not hyperexponential. But $\sin (t)^{m}$ lives in a Liouvillean field, so Kauers' Risch extension applies. 


\section{Improving Wallis' Inequality}

It is easily verified that for $n \in \mathbb{N}$ :

$$
\int_{0}^{\infty} \frac{d x}{\left(x^{2}+1\right)^{n+1}}=A(2 n) .
$$

Inserting an extra parameter $a$, Moll (11) considered the following quartic generalization of this integral,

$$
M_{n}(a):=\int_{0}^{\infty} \frac{d x}{\left(x^{4}+2 a x^{2}+1\right)^{n+1}},
$$

and presented an inspiring case study with connections to various areas of mathematics. Kauers' "extended Risch package" (7), computes

$$
\begin{gathered}
(4 n+3)(4 n+5) M_{n}(a)+8\left(a^{2}-2\right)(n+1)(2 n+3) M_{n+1}(a) \\
-16\left(a^{2}-1\right)(n+1)(m+2) M_{n+2}(a)=0 .
\end{gathered}
$$

Remark. As an alternative this recurrence could be computed with the AlmkvistZeilberger algorithm (1), since this time the integrand $\left(x^{4}+2 a x^{2}+1\right)^{-(n+1)}$ is hyperexponential.

Boros and Moll (3, Thm.7.9.1) observed that for real $a>-1$ and integer $n \geq 0$,

$$
M_{n}(a)=\frac{\pi}{2^{n+3 / 2}(a+1)^{n+1 / 2}} * P_{n}(a)
$$

where

$$
P_{n}(a)=2^{-2 n} \sum_{k=0}^{n} 2^{k}\left(\begin{array}{c}
2 n-2 k \\
n-k
\end{array}\right)\left(\begin{array}{c}
n+k \\
k
\end{array}\right)(a+1)^{k} .
$$

Having the recurrence (12) in hand, this can be easily proved using Zeilberger's algorithm (13) which computes the recurrence (12) also for the right hand side of (13).

Remark. For other proofs of (12) and (13) see (3). In (10) an alternative derivation of (13) is presented; using non-commutative Gröbner basis methods the authors derive an order 2 differential equation for $M_{n}(a)$.

Finally consider the coefficients in the expansion $P_{n}(a)=\sum_{k=0}^{n} d_{l}(n) a^{l}$ which find the sum representation

$$
d_{l}(n)=2^{-2 n} \sum_{k=l}^{n} 2^{k}\left(\begin{array}{c}
2 n-2 k \\
n-k
\end{array}\right)\left(\begin{array}{c}
n+k \\
k
\end{array}\right)\left(\begin{array}{l}
k \\
l
\end{array}\right),
$$

implied by the binomial theorem from the sum in (14). In (11) Moll introduced an interesting conjecture to a wider audience; namely,

$$
d_{l-1}(m) d_{l+1}(m) \leq d_{l}(m)^{2}, \quad 0<l<m .
$$

In other words, Moll claimed the log-concavity of the $d_{l}(m)$. In (9) M. Kauers and the first named author were able to settle Moll's conjecture by combining algorithms for deriving recurrences with G. Collins' cylindrical algebraic decomposition (CAD) (4). S. Gerhold and M. Kauers (5) were the first to introduce such a methodology for algorithmic proving of special function inequalities. Below we will show that the Gerhold-Kauers approach can be also used for automatic refinement of inequalities. 


\subsection{Proving inequalities - some remarks}

In order to illustrate the Gerhold-Kauers approach, we consider an inequality being similar to (4):

$$
\frac{1}{4 n} \leq \frac{1}{2^{4 n}}\left(\begin{array}{c}
2 n \\
n
\end{array}\right)^{2} \leq \frac{1}{3 n+1}, \quad n \geq 1
$$

Despite it can be proved automatically using Kauers' package "SumCracker" (8), an implementation of the Gerhold-Kauers method, we give a detailed proof of the right hand side inequality. The lower bound statement is proven analogously.

Proof. Denote the central term in (16) by $a(n)=2^{-4 n}\left(\begin{array}{c}2 n \\ n\end{array}\right)^{2}$. We prove the upper bound by induction, i.e., we begin by estimating

$$
a(n+1)=a(n) \frac{(2 n+1)^{2}}{(2 n+2)^{2}} \leq \frac{1}{3 n+1} \frac{(2 n+1)^{2}}{(2 n+2)^{2}},
$$

where for the last inequality we used the induction hypothesis. To complete the induction step, we need to show that for $n \geq 0$ :

$$
\frac{1}{3 n+1} \frac{(2 n+1)^{2}}{(2 n+2)^{2}} \leq \frac{1}{3 n+4}
$$

This inequality can be settled automatically using Collins' CAD, for instance, in Mathematica by executing the "Reduce" command:

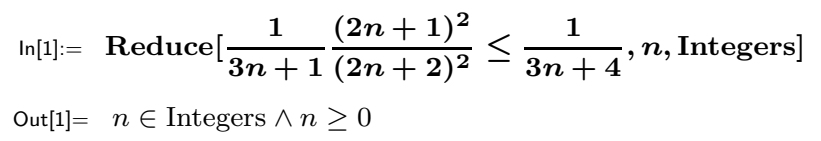

As another example let us try to prove (4) in an analogous fashion, i.e., using induction a la Gerhold-Kauers. Let $c(n)$ be as in (3), and denote the lower and upper bounds by $r_{1}(n)=\frac{2 n}{2 n+1}$ and $r_{2}(n)=1$, respectively. Setting

$$
f(n):=\frac{c(n+1)}{c(n)}
$$

for the lower estimate in the induction step of the proof one would need to show

$$
\frac{c(n+1)}{\pi}=f(n) \frac{c(n)}{\pi} \geq f(n) r_{1}(n) \geq r_{1}(n+1), \quad n \geq 0 .
$$

This last estimate, however, cannot hold in view of the fact that the sequence $c(n) / r_{1}(n)$ is monotonically decreasing towards $\pi$, i.e.,

$$
\frac{c(n+1)}{r_{1}(n+1)}<\frac{c(n)}{r_{1}(n)} \Leftrightarrow f(n)<\frac{r_{1}(n+1)}{r_{1}(n)} .
$$

This monotonicity is easily checked "by hand", and even more easily verified with CAD. Anyway, this proves that a straight-forward induction strategy a la Gerhold-Kauers is not applicable to such types of convergence estimates. On the other hand, reconsider (16) in the equivalent form

$$
\frac{2^{4 n}}{4 n}\left(\begin{array}{c}
2 n \\
n
\end{array}\right)^{-2} \leq 1 \leq \frac{2^{4 n}}{3 n+1}\left(\begin{array}{c}
2 n \\
n
\end{array}\right)^{-2}, \quad n \geq 0 .
$$


On noting that, due to (1), the latter bounds converge to $\frac{\pi}{4}<1$ and $\frac{\pi}{3}>1$, respectively, it is obvious that this is a much weaker statement than the convergence estimate in (4). Even though we stand no chance to prove Wallis' inequality by the Gerhold-Kauers procedure directly, we describe in Section 3.2 how their approach can still be used in improving the bounds in a systematic manner.

\subsection{Algorithmic refinements of Wallis' inequality}

\subsubsection{Refinements via convex combinations}

Consider the family of functions defined as convex combinations of the bounds in (4):

$$
\beta(n, \alpha):=\alpha r_{1}(n)+(1-\alpha) r_{2}(n), \quad 0<\alpha<1, \quad n \geq 0 .
$$

The goal is to determine $\underline{\alpha}(n)$ and $\bar{\alpha}(n)$ such that $r_{1}^{(1)}(n):=\beta(n, \underline{\alpha}(n))$ and $r_{2}^{(1)}(n):=$ $\beta(n, \bar{\alpha}(n))$ improve the bounds in Wallis' inequality; i.e.,

$$
r_{1}(n)<r_{1}^{(1)}(n)<\frac{c(n)}{\pi}<r_{2}^{(1)}(n)<r_{2}(n), \quad n \geq 0 .
$$

The ansatz (19) already ensures that the resulting new bounds will be between $r_{1}(n)$ and $r_{2}(n)$. It remains to compute the values for $\alpha$ that optimize the bounds within the given ansatz. For this purpose we employ CAD. To improve the lower bound in Wallis' inequality, in view of (18) we use Mathematica's "Reduce" command as follows:

$\ln [2]:=\operatorname{Reduce}\left[\left\{n \geq 0, \frac{1}{2} \leq \alpha_{0} \leq \alpha_{1}<1, \beta\left[n+1, \alpha_{1}\right] / \beta\left[n, \alpha_{0}\right] \geq f[n]\right\}\right.$, $\left\{\alpha_{0}, \alpha_{1}\right\}$, Reals]

Out[2]= $\ldots \vee\left(n \geq 1 \wedge \frac{1}{2} \leq \alpha_{0}<\frac{2 n+1}{2 n+2} \wedge \frac{4 \alpha_{0}(n+1)^{2}-2 n-1}{(2 n+1)^{2}} \leq \alpha_{1}<1\right) \vee \ldots$

The possible dependence on $n$ of the resulting function $\underline{\alpha}(n)$ is encoded by using two different $\alpha$ for CAD. Namely, the parameter $\alpha_{0}$ accounts for $\underline{\alpha}(n)$, whereas $\alpha_{1}$ corresponds to its shifted version $\underline{\alpha}(n+1)$. In the output above we omitted the expressions that do not contribute to the optimal refinement. The result of the CAD computation suggests two different values for our new bound, and it turns out that the suggested lower bound $\alpha_{0}=\frac{1}{2}$ gives the sharper, i.e., faster converging, estimate

$$
r_{1}^{(1)}(n):=\beta\left(n, \frac{1}{2}\right)=\frac{4 n+1}{4 n+2} .
$$

Observe that the suggested upper bound for $\alpha_{0}$ results in the refinement (5) given in the introduction. Correspondingly we obtain via

$\operatorname{In}[3]:=\begin{aligned} \operatorname{Reduce}\left[\left\{n \geq 0,0<\alpha_{1}<\alpha_{0} \leq \frac{1}{2}, \beta\left[n+1, \alpha_{1}\right] / \beta\left[n, \alpha_{0}\right] \leq f[n]\right\},\right. \\ \left.\left\{\alpha_{0}, \alpha_{1}\right\}, \text { Reals }\right]\end{aligned}$

the following improvement for the upper bound:

$$
r_{2}^{(1)}(n):=\beta\left(n, \frac{2 n+1}{4 n+3}\right)=\frac{4 n+2}{4 n+3} .
$$

So far we have only conjectured the bounds $r_{1}^{(1)}(n)$ and $r_{2}^{(1)}(n)$. To put things into place we summarize in the form of a theorem and give its proof. 
Theorem 4 (Refinement 1 of Wallis' inequality). Let $c(n)$ be as in (3). Then

$$
\frac{4 n+1}{4 n+2} \leq \frac{c(n)}{\pi} \leq \frac{4 n+2}{4 n+3}, \quad n \geq 0 .
$$

Proof. By construction we have that

$$
\frac{c(n)}{r_{2}(n)}<\frac{c(n)}{r_{i}^{(1)}(n)}<\frac{c(n)}{r_{1}(n)}, \quad i=1,2 .
$$

Next show (e.g., with CAD) that the sequence $c(n) / r_{1}^{(1)}(n)$ is strictly monotonically decreasing and check that indeed $c(0) / r_{1}^{(1)}(0)>\pi$.

Since $c(n) \rightarrow \pi$ and $r_{1}^{(1)}(n) \rightarrow 1$, we have $c(n) / r_{1}^{(1)}(n) \rightarrow \pi$ and in fact, because of monotonicity, $c(n) / r_{1}^{(1)}(n)$ converges to $\pi$ from above. The initial value of this sequence is greater than $\pi$, thus we must have

$$
\frac{c(n)}{r_{1}^{(1)}(n)}>\pi, \quad \text { for all } n \geq 0
$$

The second part of the Theorem is proved analogously.

Remark. The bounds in (20) are just the bounds of Gurland's refinement (6). Gurland discovered and proved these inequalities by an argument relating the variance and expectation value of certain discrete random variables.

We want to emphasize that our procedure, in principle, can be repeated arbitrarily often to further improve the estimates using the ansatz

$$
\beta^{(k)}(n, \alpha):=\alpha r_{1}^{(k)}(n)+(1-\alpha) r_{2}^{(k)}(n), \quad 0<\alpha<1, \quad n \geq 0, \quad k \geq 0,
$$

with $r_{i}^{(0)}(n):=r_{i}(n)$. In the course of these iterations the polynomial degrees of the numerators and denominators in the bounds increase. For example, the result of the next iteration step is the

Theorem 5 (Refinement 2 of Wallis' inequality). Let $c(n)$ be as in (3). Then for $n \geq 0$,

$$
r_{1}^{(2)}(n):=\frac{32 n^{2}+32 n+7}{4(2 n+1)(4 n+3)} \leq \frac{c(n)}{\pi} \leq \frac{16(n+1)(2 n+1)}{32 n^{2}+56 n+25}=: r_{2}^{(2)}(n) .
$$

\subsubsection{Refinements via extra parameters}

In Section 3.2.1 refinements of lower and upper bounds were obtained with CAD via a convex combination ansatz. Another way to invoke CAD for sharpening bounds is the clever (i.e., guided by human insight) insertion of an extra parameter. To illustrate this strategy, which in comparison to the previous CAD applications switches dependencies, we take an example from (15). Using classical methods Zhao and $\mathrm{Wu}$ derived, besides a bivariate generalization of Wallis' inequality, another improvement of Gurland's left inequality (20). For this purpose they (cleverly) introduced an extra parameter $\varepsilon$ in the lower estimate and determined for which values of $n$ this improvement holds. (Switching dependencies!) Their result (15, Thm.3.3) reads as

$$
\rho(n, \varepsilon):=\frac{8 n+2 \varepsilon+1}{8 n+2 \varepsilon-1} \frac{2 n}{2 n+1}<\frac{c(n)}{\pi}, \quad 0<\varepsilon \leq \frac{1}{2},
$$


for $n>n^{*}$, where $n^{*}$ is the maximal root of the equation

$$
32 \varepsilon n^{2}+\left(4 \varepsilon^{2}+32 \varepsilon-17\right) n+4 \varepsilon^{2}-1=0 .
$$

For $\varepsilon=\frac{1}{2}$ (and $n^{*}=0$ ) this corresponds to Gurland's bound (20). As $\varepsilon$ approaches zero, $n^{*}$ tends to infinity. The lower bound $n^{*}$ can be chosen as the position from which on the sequence approximating $\pi$ becomes monotonically decreasing. Certainly this task can be formulated in a way such that CAD is again applicable to determine the lower bound $n^{*}$ as follows:

$\operatorname{In}[4]:=\operatorname{Reduce}\left[\left\{0<\varepsilon \leq \frac{1}{2}, \frac{\rho[n+1, \varepsilon]}{\rho[n, \varepsilon]} \geq f[n]\right\},\{n\}\right.$, Reals $]$

The first condition in the argument of "Reduce" imposes that the sequence $c(n) / \rho(n, \varepsilon)$ has to be monotonically decreasing. In short, the CAD computation yields the bound

$$
n^{*}=\frac{-4 \varepsilon^{2}-16 \varepsilon+9}{32 \varepsilon},
$$

which turns out to be an improvement over the bound given in (15). Again, it only remains to check that $c\left(n_{0}\right) / \rho\left(n_{0}, \varepsilon\right)$ is greater than $\pi$ for some integer $n_{0} \geq n^{*}$.

In (15) Zhao and Wu did not consider an $\varepsilon$-improvement of the upper bound in Gurland's inequality (20). However, such a bound can be obtained easily with our CAD strategy. To this end we introduce the extra parameter $\varepsilon$ in the upper bound as

$$
\rho(n, \varepsilon):=\frac{4 n+2-\varepsilon}{4 n+3-\varepsilon} .
$$

Note that with this definition we have $\rho(n, 0)=r_{2}^{(1)}(n)$, and also $\rho(n, 1)=r_{1}^{(1)}(n)$. The condition for improving the upper bound is that the sequence $\rho(n, \varepsilon)$ needs to be monotonically increasing:

$\operatorname{In}[5]:=\operatorname{Reduce}\left[\left\{0<\varepsilon \leq \frac{1}{2}, \frac{\rho[n+1, \varepsilon]}{\rho[n, \varepsilon]} \leq f[n]\right\},\{n\}\right.$, Reals $]$

This computation yields for $n$ the lower bound $n^{*}=\frac{2-9 \varepsilon+\varepsilon^{2}}{8 \varepsilon-4}$, which tends to infinity as $\varepsilon \rightarrow \frac{1}{2}$. It is interesting to note that for this limit value we obtain an improved lower bound for $c(n) / \pi$, namely $\rho\left(n, \frac{1}{2}\right)=\frac{8 n+3}{8 n+5} \geq \frac{4 n+1}{4 n+2}=r_{1}^{(1)}(n)$.

\section{Conclusion}

We expect that our variation of the Gerhold-Kauers approach illustrated in Section 3.2 has a much wider range of applicability. In particular, inequalities involving more than one discrete parameter should provide an even more promising application domain. For instance, in the proof (9) of Moll's log-concavity conjecture (9) recurrences in $d_{l}(m)$ involving both parameters, $l$ and $m$, played a crucial role. In contrast, the examples we gave above involved just one discrete parameter and recurrences of order 1 only. Consequently the CAD steps remained on elementary grounds, mostly imposing monotonicity conditions. 


\section{References}

[1] G. Almkvist and D. Zeilberger, The method of differentiating under the integral sign, J. Symb. Comp. 10 (1990), 571-591.

[2] G.E. Andrews, R. Askey, and R. Roy, Special Functions, Cambridge University Press, 1999.

[3] G. Boros and V.H. Moll, Irresistible Integrals, Cambridge University Press, 2004.

[4] G.E. Collins, Quantifier elimination for the elementary theory of real closed fields by cylindrical algebraic decomposition, Lecture Notes in Computer Science 33 (1975), 134-183.

[5] S. Gerhold and M. Kauers, A procedure for proving special function inequalities involving a discrete parameter, In: Proceedings of ISSAC'05 (2005), 156-162.

[6] J. Gurland, On Wallis' formula, Amer. Math. Monthly 63 (1956), 643-645.

[7] M. Kauers, An experimental implementation of the Risch integration procedure, Mathematica package for internal use, RISC, J. Kepler University, 2006.

[8] M. Kauers, SumCracker: A package for manipulating symbolic sums and related objects, J. Symb. Comput. 41 (2006), 1039-1057,

[9] M. Kauers and P. Paule, A computer proof of Moll's log-concavity conjecture, Proc. Amer. Math. Soc. 135 (2007), 3847-3856.

[10] C. Koutschan and V. Levandovsky, Computing one of Victor Moll's irresistable integrals with computer algebra, Comput. Sci. J. Moldova 16(1) (2008), 35-49.

[11] V. Moll, The evaluation of integrals: A personal story, Notices Amer. Math. Soc. 49 (2002), 311-317.

[12] J. Wallis, Arithmetica Infinitorum, Oxford, 1656.

[13] D. Zeilberger, A fast algorithm for proving terminating hypergeometric identities, Discrete Math. 80 (1982), 207-211.

[14] D. Zeilberger, The method of creative telescoping, J. Symb. Comp. 11 (1991), 195204.

[15] Y. Zhao and Q. Wu, Wallis inequality with a parameter, J. Inequal. Pure Appl. Math. 7(2) (2006), 10 pp., electronic. 